\title{
High-frequency ultrasonography of psoriatic skin: A non-invasive technique in the evaluation of the entire skin of patients with psoriasis: A pilot study
}

\author{
MIHAELA CRISTINA ȘOMLEA ${ }^{1}$, ANDREEA NICOLETA BOCA ${ }^{2}$, ALEXANDRA DANA POP $^{3}$, \\ ROXANA FLAVIA ILIEȘ ${ }^{4}$, STEFAN CRISTIAN VESA ${ }^{2}$, ANCA DANA BUZOIANU $^{2}$ and ALEXANDRU TĂTARU ${ }^{1}$ \\ ${ }^{1}$ Department of Dermatology, 'Iuliu Hațieganu' University of Medicine and Pharmacy, 400006 Cluj-Napoca; \\ ${ }^{2}$ Department of Pharmacology, Toxicology and Clinical Pharmacology, 'Iuliu Hațieganu' University \\ of Medicine and Pharmacy, 400337 Cluj-Napoca; ${ }^{3}$ Department of Physiology, \\ 'Iuliu Hațieganu' University of Medicine and Pharmacy, 400006 Cluj-Napoca; ${ }^{4}$ Department of Medical Genetics, \\ 'Iuliu Hațieganu’ University of Medicine and Pharmacy, 400212 Cluj-Napoca, Romania
}

Received September 11, 2018; Accepted March 6, 2019

DOI: $10.3892 /$ etm.2019.8140

\begin{abstract}
Psoriasis is a chronic, systemic, inflammatory disorder which accelerates the life process of skin cells, based on a genetically induced deviant immune response. High-frequency ultrasonography (HF-USG) is a painless, non-invasive imaging technique that can be performed and repeated whenever the need arises. We evaluated lesional and non-lesional skin of psoriatic patients with the use of HF-USG, focusing on the immune-induced inflammation and skin thickness. Previous studies suggested that HF-USG, being a non-invasive technique, is useful as an aid to clinical evaluation of the severity of psoriatic plaques. Our goal was to determine whether the skin of psoriatic patients is influenced by the background or habits of the patients. The study included a total of 27 patients affected by psoriasis vulgaris. The thickness of the epidermis and dermis and the skin echogenicity were documented for the active plaques, as well as for the non-affected skin of all the patients included in the study, using a high-frequency ultrasonographic system. The patient's local background, sex, family history of psoriasis, smoking habits and sun exposure were analyzed. HF-USG of the psoriatic plaques exposes a three-band structure that is easily distinguished from the surrounding unaffected skin,
\end{abstract}

Correspondence to: Dr Andreea Nicoleta Boca, Department of Pharmacology, Toxicology and Clinical Pharmacology, 'Iuliu Hațieganu' University of Medicine and Pharmacy, 16 Rahovei Street, 400337 Cluj-Napoca, Romania

E-mail: andreea.boca@hotmail.com

Abbreviations: HFUSG, high-frequency ultrasonography; SLEB, subepidermal low-echogenic band; PASI, Psoriasis Area and Severity Index

Key words: psoriasis, skin ultrasonography, thickness, immune induced, inflammation due to a hypoechoic band in the upper dermis. Although not specific for psoriasis, it is a strong marker of inflammation. The obtained results confirm that, indeed, skin thickness is greater in lesional skin compared to non-lesional skin, by a mean of $1,180 \mu \mathrm{m}( \pm 340 \mu \mathrm{m})$. We consider that skin HF-USG should be used as a quantitative method in the clinical evaluation of the patients with psoriasis and may help as an objective means of assessing inflammation in lesional skin.

\section{Introduction}

Psoriasis is a chronic, systemic, non-infectious, inflammatory disease, easily recognized by its clinical appearance (1). Inflammation, which is one of the weapons used by the immune system to fight an intruder, occurs at skin level, resulting in inflammation and thickening of the skin. This manifests clinically as patches of erythematous skin, covered in adherent, silvery scales. A genetic component to psoriasis has been identified; psoriasis is considered a multifactorial condition, where both the genetic predisposition and the environmental triggers (infections, certain medications, mental distress) must occur in a patient to lead to a clinical manifestation of the condition $(2,3)$.

The disorder affects women and men of all ages, all nations, with a known prevalence of 2-3\% in Western countries $(2,4,5)$. The skin of the psoriasis plaques appears firmer and thickened in contrast to the skin that surrounds the lesion (1). This has been proved histologically in accordance to standard medical literature: until 1980, the emphasis was on alteration of keratinocyte proliferation and differentiation (6). The main changes of skin histology are hyper-proliferation of keratinocytes in the intra-follicular epidermis and epidermal rete, which become elongated and descend into the dermis (2). The other alteration is related to vasodilatation developing enlarged and tortuous capillary circumferences in the dermis. Polymorphonuclear leucocytes and T lymphocytes, particularly Th1-type $\mathrm{T}$ cells [which produce interferon- $\gamma$ $($ IFN- $\gamma)]$, represent the inflammatory cell infiltration of the upper dermis (1). The differentiation of keratinocytes is 
widely modified in psoriasis. The accelerated process of hyper-proliferation can be distinguished as the 'regenerative maturation', similar to wound repair (5). Nowadays, studies emphasize that psoriasis seems to be a product of faulty interaction among the environmental factors, the epidermal cell which produces keratin and immune cells, leading to the activation of pro-inflammatory cytokines, such as IFN- $\gamma$, tumour necrosis factor (TNF), interleukin-1 (IL-1), IL-6, IL-17, IL-22, chemokines-activated leukocytes and T-cells. These modulate a continuous inflammatory process in the skin, which is fully reversible with appropriate therapy $(7,8)$. Moreover, it is one of the most common chronic cutaneous diseases involving Th-1, Th17 and Th-22. This leads to the exacerbated multiplication of T-cells mentioned previously (9). Although it is an organ-specific autoimmune disease, like Crohn's disease, rheumatoid arthritis, multiple sclerosis and juvenile-onset diabetes, psoriasis often associates with other systemic pathologies, such as cardiovascular disorders or metabolic syndrome. Concomitance of psoriasis and atopic dermatitis is relatively very rare, but it is invariably under discussion whether these disorders are immunopathology connected.

Determining histological changes in the traditional method, using a biopsy, is an invasive procedure, involving some risks to the patient (10). As such, this cannot be performed regularly for assessing the inflammatory state in disease evolution. Therefore, a non-invasive procedure is required for quantifying skin changes in patients with psoriasis (11). High-frequency ultrasound has brought forth a potential solution for monitoring cutaneous alteration. High-frequency ultrasonography (HF-USG) is a non-invasive method, offering objective measurements in real-time; it seems to fulfill the need for more accurate monitoring in psoriasis patients. Furthermore, there are no contraindications or limitations for applying this procedure $(12,13)$.

Even though the histopathological and immunological background of psoriasis has been well detailed, the diagnosis of psoriasis is a clinical one, relying on the sites of predilection in which the lesions arise: knees, elbows, lower back and scalp. The severity of the disease is mainly measured with the help of a clinical scoring system such as the Psoriasis Area and Severity Index (PASI), which integrates the severity of the erythema, induration, desquamation and percentage of affected area or Dermatology life Quality Index (DLQI), which quantifies the patients' quality of life $(14,15)$. Clinical scores are a subjective measurement and there are no absolute criteria to appreciate, for instance, how red, thick or rough a plaque of psoriasis is. The assessment depends on the specialist's expertise bringing forward the issue of observer bias in applying the current clinical scores. The present study discusses the degree of inflammation in lesional skin compared to nonlesional skin, correlating the parameters obtained by HF-USG with potential trigger or risk factors such as smoking, sun exposure, treatment compliance, as well as unmodifiable factors (ethnicity, sex, and genetic traits).

\section{Materials and methods}

Patients. Twenty-seven patients with early onset disease or disease uncontrolled by conventional therapy were selected. The patients were questioned about age, sex, duration of
Table I. The results of the imaging tests of the injured skin, as compared to the adjacent healthy skin.

\begin{tabular}{lllc}
\hline Variables & $\begin{array}{c}\text { Psoriatic } \\
\text { lesion }\end{array}$ & \multicolumn{1}{c}{$\begin{array}{c}\text { Healthy } \\
\text { skin }\end{array}$} & P-value \\
\hline Skin thickness $(\mu \mathrm{m})$ & $1.180 \pm 340$ & $1.101 \pm 269$ & 0.03 \\
Intensity $(\mu \mathrm{m})$ & $25.81 \pm 12.03$ & $64.63 \pm 30.86$ & 0.01 \\
\hline
\end{tabular}

disease, home environment, PASI score, history of psoriasis in their family, smoking habits and exposure to sun. Eight men $(29.6 \%)$ and 19 women $(70.4 \%)$ with active psoriasis plaques, without other associated pathologies were enrolled in this study. Measurements were performed before initiating or prior to updating psoriasis therapy. All subjects had received histological diagnosis confirming psoriasis vulgaris. Patients who did not have a positive histological diagnosis of psoriasis were excluded from the study.

The protocol and acceptance form were approved by the Ethics Committee of University of Medicine and Pharmacology 'Iuliu Hatieganu' (Cluj-Napoca, Romania), and all patients gave an informed consent after a complete discussion of the procedures, techniques, applicability and benefits of the study.

Methods. The clinical severity of the psoriasis lesions was assessed using the PASI score, based on the extent of the lesion and its characteristics (erythema, thickness and scaling). We considered a PASI score under 10 to qualify as mild psoriasis, a score of 11 to 20 as moderate psoriasis and a score above 20 as severe psoriasis. One plaque which was representative for the subjects' lesions was chosen by the physician, on either the arms, calves, posterior thorax or abdomen of the patient. For each plaque, an apparently healthy, adjacent skin area was chosen to compare ultrasound images.

Skin ultrasound. The skin's examinations were performed using the DermaLab Skin analysis technology. The images were obtained manipulating a B-mode high-resolution ultrasound system using a $20-\mathrm{MHz}$ center frequency transducer that supports acquired cross-sectional images of the skin up to $2.5 \mathrm{~cm}$ in depth. A drop of water was placed on the transductor, as a conductive medium, the resolution of the images being $60 \times 200 \mu \mathrm{m}$. The transducer was positioned perpendicular to the longitudinal axis of the skin, in the center of the psoriasis plaque and compared closely to healthy skin of the same individual. For every patient, the images were analyzed by studying the subepidermal low-echogenic band (SLEB), collagen levels, dermal thickness and echo-intensity. The thickness of the dermal skin was calculated in micrometers based on a super A scan. Additionally, this scan type indicates the echo intensity, referring to the scale of skin damage. The SLEB thickness is measured in micrometers, related to inflammatory process and edema of the skin.

Statistical analysis. Statistical analysis was performed using the MedCalc Statistical Software (version 18.5; MedCalc Software BVBA, Ostend, Belgium). Continuous data are expressed using mean and standard deviation (mean $\pm \mathrm{SD}$ ), and nominal data are 
Table II. The ultrasonography results of the correlations between the imaging tests and sex.

\begin{tabular}{|c|c|c|c|c|c|}
\hline \multirow[b]{3}{*}{ Variables } & \multicolumn{4}{|c|}{ Sex } & \multirow[b]{3}{*}{ P-value } \\
\hline & \multicolumn{2}{|c|}{ Male } & \multicolumn{2}{|c|}{ Female } & \\
\hline & Lesions & Healthy skin & Lesions & Healthy skin & \\
\hline Skin thickness $(\mu \mathrm{m})$ & $1.343 \pm 424$ & $952 \pm 368$ & $1111.3 \pm 284.1$ & $1.022 \pm 224.1$ & 0.1 \\
\hline Intensity $(\mu \mathrm{m})$ & $30.1 \pm 19.6$ & $81.9 \pm 29.18$ & $24 \pm 6.9$ & $57.34 \pm 29.25$ & 0.4 \\
\hline $\operatorname{SLEB}(\mu \mathrm{m})$ & $238.25 \pm 99.22$ & 0 & $239.58 \pm 101.64$ & 0 & 0.9 \\
\hline
\end{tabular}

SLEB, sub-epidermal low echogenic band.

Table III. The results regarding the correlation between the skin and home environment.

\begin{tabular}{|c|c|c|c|c|c|}
\hline \multirow[b]{3}{*}{ Variables } & \multicolumn{4}{|c|}{ Home environment } & \multirow[b]{3}{*}{ P-value } \\
\hline & \multicolumn{2}{|c|}{ Urban } & \multicolumn{2}{|c|}{ Rural } & \\
\hline & Lesions & Healthy skin & Lesions & Healthy skin & \\
\hline Skin thickness $(\mu \mathrm{m})$ & $1216.6 \pm 383.94$ & $998.84 \pm 319$ & $1.092 \pm 199.5$ & $1007.5 \pm 75.25$ & 0.2 \\
\hline Intensity $(\mu \mathrm{m})$ & $24.21 \pm 12.64$ & $69.21 \pm 33.25$ & $29.62 \pm 10.14$ & $53.76 \pm 22.44$ & 0.2 \\
\hline $\operatorname{SLEB}(\mu \mathrm{m})$ & $229.68 \pm 109.59$ & 0 & $261.75 \pm 68.55$ & 0 & 0.4 \\
\hline
\end{tabular}

SLEB, subepidermal low-echogenic band.

characterized by absolute and relative frequency. Comparison between groups was carried out using Student's t-test and ANOVA followed by Tukey's post hoc test, whenever appropriate. Correlations between quantitative variables, like PASI clinical score or ultrasonography measurements, were verified using Pearson's correlation. P-value $<0.05$ was considered to indicate a statistically significant difference.

\section{Results}

The patients' age ranged between 11 and 62 years, with a mean of 35.89 years $( \pm 13.754)$. The average age of onset was 22 years, with variations ranging from 1 to 40 years. Current PASI score ranged from 3.6 to 36.2. The ultrasonographic acquisitions for lesional and nonlesional skin are available upon demand.

In 3 volunteer patients aged over 40, the SLEB was also present in the nonlesional images, as a consequence of skin aging. In few cases, undulation of the epidermis could be detected. The thickness of the skin in the entire group presenting psoriasis plaques was increased by a mean of $1,180 \pm 340 \mu \mathrm{m}$ as compared to healthy skin, with an average of $16 \%$ (1.180 vs. $1.001 \mu \mathrm{m})$, while intensity on healthy skin was higher, with a percentage of $50 \%$ compared to normal skin.

Table I includes the full ultrasonographic parameters obtained; using Student's t-test, it was noted that skin thickness and skin intensity are strongly determined by the presence of the psoriasis lesion, with a $\mathrm{P}=0.038$, respectively 0.001 for the population tested. Using the same test, we observed that there is no statistical correlation between age and SLEB, skin thickness and intensity for lesional skin or nonlesional skin.

Table II shows the correlations obtained between imaging parameters and sex. Addressing differences based on sex, the following were noted: For women lesional skin thickness is $21 \%$ lower than for men, while on nonlesional skin it appears $3 \%$ lower for women than for men. Intensity on lesional skin is $25 \%$ lower for women than for men, while on nonlesional areas it is $43 \%$ lower. With regards to the lesional images, the SLEB is similar for men vs. women, with a difference of $1 \%$ in favour of women. Applying Student's t-test, it is noted that skin thickness and intensity on plaques are influenced by the sex of the patient, while SLEB is not influenced. This can be explained due to the aftermath of hormonal influence, especially after menopause or ovariectomies, as decreased estrogen levels result in thinned skin, while estrogen therapy thickens the skin.

In respect to any differences based on home location (rural vs. urban), we noted that injured skin thickness is $10 \%$ higher for patients living in urban areas, whereas healthy, uninjured skin presents almost similar thickness, $1 \%$ in favor of the patients living in urban areas, regardless of the home environment of the patient. Intensity on affected skin is $22 \%$ higher in rural versus urban patients, while on healthy skin intensity is higher for urban patients compared to rural ones. SLEB is $14 \%$ higher for rural patients vs. urban ones; this was also confirmed by applying Student's t-test. The full parameters obtained are depicted in Table III.

When dealing with family history of psoriasis, we noted that those with family history are predisposed to having 
Table IV. The results of the correlations assumed between the imaging tests and family history of patients diagnosed with psoriasis.

Psoriasis family history

\begin{tabular}{|c|c|c|c|c|c|}
\hline \multirow[b]{2}{*}{ Variables } & \multicolumn{2}{|c|}{ Affirmative } & \multicolumn{2}{|c|}{ Negative } & \multirow[b]{2}{*}{ P-value } \\
\hline & Lesions & Healthy skin & Lesions & Healthy skin & \\
\hline Skin thickness $(\mu \mathrm{m})$ & $1045.91 \pm 219.91$ & $978.82 \pm 86.93$ & $1272.19 \pm 383$ & $1016.94 \pm 346$ & 0.06 \\
\hline Intensity $(\mu \mathrm{m})$ & $25.87 \pm 10.37$ & $51.38 \pm 25.2$ & $25.77 \pm 13.38$ & $73.75 \pm 31.78$ & 0.9 \\
\hline $\operatorname{SLEB}(\mu \mathrm{m})$ & $241.45 \pm 69.72$ & 0 & $237.63 \pm 117$ & 0 & 0.9 \\
\hline
\end{tabular}

SLEB, sub-epidermal low echogenic band.

Table V. Correlation between smoking and noted variables.

\begin{tabular}{|c|c|c|c|c|c|}
\hline \multirow[b]{3}{*}{ Variables } & \multicolumn{4}{|c|}{ Smoking habit } & \multirow[b]{3}{*}{ P-value } \\
\hline & \multicolumn{2}{|c|}{ Affirmative } & \multicolumn{2}{|c|}{ Negative } & \\
\hline & Lesions & Healthy skin & Lesions & Healthy skin & \\
\hline Skin thickness $(\mu \mathrm{m})$ & $1280.25 \pm 275.08$ & $984.25 \pm 156.78$ & $1162.57 \pm 353.17$ & $1004.39 \pm 284.65$ & 0.5 \\
\hline Intensity $(\mu \mathrm{m})$ & $14.87 \pm 3.05$ & $68.22 \pm 38.52$ & $27.71 \pm 12.01$ & $64.01 \pm 30.34$ & 0.01 \\
\hline SLEB $(\mu \mathrm{m})$ & $220.25 \pm 64.59$ & 0 & $242.48 \pm 104.59$ & 0 & 0.6 \\
\hline
\end{tabular}

SLEB, sub-epidermal low echogenic band.

thinner affected and healthy skin. The full results are available in Table IV.

Considering the influence of smoking, we noted that skin thickness on lesional areas is $10 \%$ higher for smokers, while healthy skin is $3 \%$ thicker for non-smokers than for smokers. Echo-intensity for affected areas is $46 \%$ higher for non-smokers, while for healthy skin it is $6 \%$ lower, as opposed to smokers'. SLEB registered higher values by $10 \%$ in the non-smoker group (Table V).

The influence of sun exposure results showed that skin thickness on lesional areas is 7\% higher for those exposed, while healthy skin is $16 \%$ thicker for those exposed. Intensity on affected skin is $22 \%$ higher for those who are not exposed to the sun, while for healthy skin it is only $19 \%$ higher. SLEB is $12 \%$ higher for those who expose themselves to the sun versus those who do not (Table VI).

Correlating treatment compliance and ultrasound measurements, it was observed that skin on affected areas is $3 \%$ thinner for those who undergo treatment and the intensity of lesional areas is $30 \%$ higher for those that do not. SLEB is $7 \%$ higher for those that are subjected to treatment, but this cannot be considered relevant, since the patients involved in this study did not follow proper treatment, therefore their psoriasis was uncontrolled, or the affection presented itself at its onset (Table VII).

Patients that underwent biological therapy had thicker healthy skin, compared with those who did not $(\mathrm{P}=0.025)$. Injured skin thickness was lower in patients with biological therapy, without statistical significance $(\mathrm{P}=0.2)$. Intensity on affected skin was higher in patients with biological therapy, without statistical significance $(\mathrm{P}=0.4)$. SLEB was higher in patients with biological therapy, without statistical significance $(\mathrm{P}=0.7)$. No correlation between the PASI clinical score and the ultrasonography measurements of the thickness of the skin was found.

\section{Discussion}

Compared to healthy skin, psoriasis plaques display distinctive alterations of the skin layers, produced by underlying inflammation $(16,17)$. Overall, in all examined psoriasis plaques, skin thickness was increased, and the echo-intensity was lower, in contrast to the adjacent skin (17-19). Many researchers have observed that there is a highly significant increase in the thickness of the affected skin of patients with psoriasis. El Gammal et al (5) considered that this is due to the papillary dermis which fluctuates in length due to inflammation, oedema and vascularity variation. The thickness of the epidermal layer may be caused by the keratocyte proliferation and desquamation (5). The increase of the dermal component may be a result of leucocyte infiltration and epidermal acanthosis and the SLEB variation may be due to erythema, which is an inflammatory process caused by fluid fluctuation, but it is also possible that SLEB also reflects acanthosis.

The skin's oedema, determined by the presence of water leads to restructuring of the dermis, by increasing the density between collagen fibers, which can be an explanation for decreasing skin consistency (17). 
Table VI. The values obtain after correlating with sun exposure.

\begin{tabular}{|c|c|c|c|c|c|}
\hline \multirow[b]{3}{*}{ Variables } & \multicolumn{4}{|c|}{ Sun exposure } & \multirow[b]{3}{*}{ P-value } \\
\hline & \multicolumn{2}{|c|}{ Affirmative } & \multicolumn{2}{|c|}{ Negative } & \\
\hline & Lesions & Healthy skin & Lesions & Healthy skin & \\
\hline Skin thickness $(\mu \mathrm{m})$ & $1226.67 \pm 399.79$ & $1121.33 \pm 254.25$ & $1142.67 \pm 294.36$ & $905.47 \pm 248$ & 0.5 \\
\hline Intensity $(\mu \mathrm{m})$ & $23 \pm 9.6$ & $58.45 \pm 24.85$ & $28 \pm 13.55$ & $69.58 \pm 35$ & 0.2 \\
\hline $\operatorname{SLEB}(\mu \mathrm{m})$ & $253.5 \pm 101.4$ & 0 & $227.73 \pm 99$ & 0 & 0.5 \\
\hline
\end{tabular}

SLEB, sub-epidermal low echogenic band.

Table VII. The results of the correlations assumed between the imaging tests and patient compliance in treatment.

\begin{tabular}{|c|c|c|c|c|c|}
\hline \multirow[b]{3}{*}{ Variables } & \multicolumn{4}{|c|}{ Treatment compliance } & \multirow[b]{3}{*}{ P-value } \\
\hline & \multicolumn{2}{|c|}{ Affirmative } & \multicolumn{2}{|c|}{ Negative } & \\
\hline & Lesions & Healthy skin & Lesions & Healthy skin & \\
\hline Skin thickness $(\mu \mathrm{m})$ & $1168.13 \pm 305.36$ & $932.27 \pm 263.9$ & $1194.83 \pm 394.06$ & $1.087 \pm 260.47$ & 0.8 \\
\hline Intensity $(\mu \mathrm{m})$ & $22.86 \pm 8.79$ & $64.76 \pm 30.02$ & $29.5 \pm 14.73$ & $64.47 \pm 33.23$ & 0.1 \\
\hline SLEB $(\mu \mathrm{m})$ & $246.2 \pm 118.2$ & 0 & $230.4 \pm 72.1$ & 0 & 0.6 \\
\hline
\end{tabular}

SLEB, sub-epidermal low echogenic band.

Based on our results we concluded that the skin thickness is lower in women than in men. This can be attributed to hormonal influence, postmenopausal or post ovariectomy, decreased estrogen levels lead to thinning of the skin, while estrogen therapy thickens the skin. When analyzing their background, we observed that the patients living in urban areas display thickened lesional skin, as well as thickened adjacent healthy skin.

The thickened healthy skin may be explained by the lack of sun exposure. As for patients residing in rural areas, we expect a higher rate of sun exposure. This, in turn, lowers inflammation present in psoriasis lesions, but, at the same time, determines premature skin aging and, thus, cutaneous thinning. The values obtained in this study have demonstrated that, indeed, those who are more exposed to the sun for a longer period have thinner skin.

For patients with a positive family history of psoriasis, the entire skin, both healthy and affected, is thinner, comparing to the skin of those who do not have this genetic background. This result supports the theory of genetic immune induced transmission of the disease. In the case of psoriasis, familial incidence can be easily proven and at times can be followed throughout the history of several generations.

The increase of the SLEB in psoriasis lesions is an accepted, demonstrated fact, showing the degree of inflammation. Even if SLEB increases during the ultrasound examination of psoriasis lesions, it is not a specific marker for psoriasis (18-20). SLEB is also known as 'solar elastoic band', being a marker of tegument aging, seen in the damaged sun exposed skin. The SLEB values obtained in this study did not differ when it comes to sex, but it is slightly variant according to the patient's environmental background, as in these cases, the SLEB values is higher, due to regular sun exposure $(19,21)$.

In conclusion, an ultrasound system which uses high-frequency $(20 \mathrm{MHz})$ can provide relevant information regarding the consequences of skin inflammation in psoriasis. The increased thickness, as well as the level of inflammation may be quantified using HF-USG in order to better monitor patients' evolution under therapy. The ultrasonographic image of a psoriasis lesion shows increased skin thickness, lowered echointensity and an increase in SLEB.

\section{Acknowledgements}

The authors would like to thank all the participants and the medical staff, who helped during data collection.

\section{Funding}

No funding was received.

\section{Availability of data and materials}

The datasets used and/or analyzed during the current study are available from the corresponding author on reasonable request. 


\section{Author's contributions}

MCS, ANB, RFI, ADP, SCV, ADB and AT contributed to the design of the study, acquisition of the data, and researched the data and worked on interpretation of the results. ANB prepared the questionnaire and obtained the images. SCV performed the statistical analysis. MCS, ANB and ADP contributed to the writing of the manuscript. AT is the guarantor of this work and, as such, had full access to all the data in the study and takes responsibility for the integrity of the data and the accuracy of the data analysis. All authors read and approved the final version of the manuscript.

\section{Ethics approval and consent to participate}

The present study was carried out following the approval of the University's Ethics Board, in accordance to the principles of the Helsinki Declaration. The patients were provided with all the necessary explanations regarding the purpose of the study and their involvement in it, and signed informed consent forms to participate in this study.

\section{Patient consent for publication}

Patient consent for publication was obtained on the same form as the informed consent for participating in the study.

\section{Competing interests}

The authors declare that they have no competing interests.

\section{References}

1. Burgdorf W, Plewig G, Wolff HH and Landthaler M (eds): Psoriasis. In: Braun Falco's Dermatology. 3rd edition, Springer-Verlag, Berlin, pp506-508, 2009.

2. Rocken M, Schaller M, Sattler E and Bargdorf W: Color Atlas of Dermatology. Yale J Biol Med 85: 430-431, 2012.

3. Bolognia J, Schaffer J, Duncan K and Ko C: Dermatology Essentials. Saunders/Elsevier, Philadelphia, 2014.

4. Parisi R, Symmons DP, Griffiths CE and Ashcroft DM; Identification and Management of Psoriasis and As-sociated ComorbidiTy (IMPACT) project team: Global epidemiology of psoriasis: A systematic review of incidence and prevalence. J Invest Dermatol 133: 377-385, 2013.

5. El Gammal S, El Gammal C, Kaspar K, Pieck C, Altmeyer P, Vogt $\mathrm{M}$ and Ermert H: Sonography of the skin at $100 \mathrm{MHz}$ enables in vivo visualization of stratum corneum and viable epidermis in palmar skin and psoriatic plaques. J Invest Dermatol 113: 821-829, 1999
6. Wolff K, Johnson RA and Saavedra AP (eds): Fitzpatrick's Color Atlas and Synopsis of Clinical Dermatology. 7th edition. McGraw Hill, New York, pp52-56, 2013.

7. Boda D, Negrei C, Nicolescu F and Bălălău C: Assessment of some oxidative stress parameters in methotrexate treated psoriasis patients. Farmacia 62: 704-710, 2014

8. Caruntu C, Boda D, Dumitrascu G, Constantin C and Neagu M: Proteomics focusing on immune markers in psoriatic arthritis. Biomarkers Med 9: 513-528, 2015.

9. Furue K, Ito T, Tsuji G, Kadono T, Nakahara T and Furue M: Autoimmunity and autoimmune co-morbidities in psoriasis. Immunology 154: 21-27, 2018.

10. Batani A, Brănișteanu DE, Ilie MA, Boda D, Ianosi S, Ianosi G and Caruntu C: Assessment of dermal papillary and microvascular parameters in psoriasis vulgaris using in vivo reflectance confocal microscopy. Exp Ther Med 15: 1241-1246, 2018.

11. Căruntu C, Boda D, Căruntu A, Rotaru M, Baderca F and Zurac S: In vivo imaging techniques for psoriatic lesions. Rom J Morphol Embryol 55 (Suppl 3): 1191-1196, 2014.

12. Vaillant L, Berson M, Machet L, Callens A, Pourcelot L and Lorette G: Ultrasound imaging of psoriatic skin: A noninvasive technique to evaluate treatment of psoriasis. Int J Dermatol 33: 786-790, 1994.

13. Gutierrez M, Wortsman X, Filippucci E, De Angelis R, Filosa G and Grassi W: High-frequency sonography in the evaluation of psoriasis: Nail and skin involvement. J Ultrasound Med 28: 1569-1574, 2009.

14. Gupta AK, Turnbull DH, Harasiewicz KA, Shum DT, Watteel GN, Foster FS and Sauder DN: The use of high-frequency ultrasound as a method of assessing the severity of a plaque of psoriasis. Arch Dermatol 132: 658-662, 1996.

15. Polańska A, Dańczak-Pazdrowska A, Jałowska M, Żaba R and Adamski Z: Current applications of high-frequency ultrasonography in dermatology. Postepy Dermatol Alergol 34: 535-542, 2017.

16. Cucoş M, Crişan M, Lenghel M, Dudea M, Croitoru R and Dudea SM: Conventional ultrasonography and sono-elastography in the assessment of plaque psoriasis under topical corticosteroid treatment - work in progress. Med Ultrason 16: 107-113, 2014.

17. Marina ME, Botar Jid C, Roman II, Mihu CM and Tătaru AD: Ultrasonography in psoriatic disease. Med Ultrason 17: 377-382, 2015.

18. Shenoy A: High resolution ultrasonography in dermatology; a psoriasis experience. Indian J Basic Appl Med Res 5: 121-125, 2016.

19. Crisan D, Lupsor M, Boca A, Crisan M and Badea R: Ultrasonographic assessment of skin structure according to age. Indian J Dermatol Venereol Leprol 78: 519, 2012.

20. Gniadecka M, Gniadecki R, Serup J and Søndergaard J: Ultrasound structure and digital image analysis of the subepidermal low echogenic band in aged human skin: Diurnal changes and interindividual variability. J Invest Dermatol 102: 362-365, 1994.

21. Serup J: Non-invasive quantification of psoriasis plaques - measurement of skin thickness with $15 \mathrm{mHz}$ pulsed ultrasound. Clin Exp Dermatol 9: 502-508, 1984. 